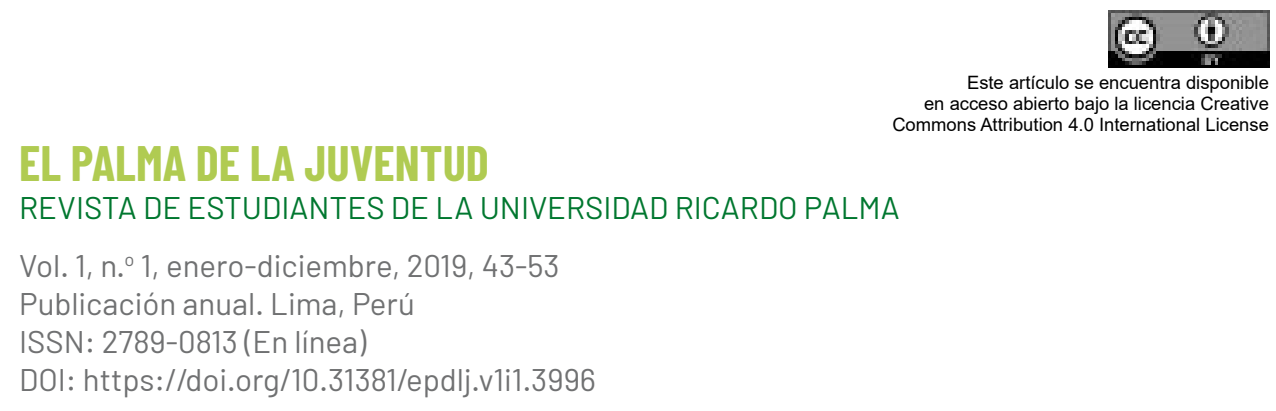

\title{
LA FIGURA DEL AROUITECTO Y SU IDEOLOGÍA AL PIE DE LA LETRA EN LAS TRADICIONES PERUANAS DE RICARDO PALMA'
}

\section{The figure of the architect and his literal ideology in Tradiciones peruanas by Ricardo Palma}

ANDREA YOAN ROMUCHO ATAUQUU

Facultad de Arquitectura y Urbanismo, Universidad Ricardo Palma Lima, Perú

Contacto: andrea.romucho@urp.edu.pe

\section{RESUMEN}

Este artículo abordará las tradiciones «Lavaplatos»y «Al pie de la letra». Se propone que en estos textos el tradicionalista satiriza la figura del arquitecto y sus ideologías estancadas.

Palabras clave: Tradiciones peruanas; arquitectura; ideología.

1 Este artículo se elaboró como parte del curso Taller de Comunicación Oral y Escrita II, asignatura dictada por la profesora Vilma Vera Collazos en el semestre 2019-I. 


\section{ABSTRACT}

This article will discuss the traditions «Lavaplatos» and «Al pie de la letra». In these texts, it is proposed that the traditionalist satirizes the figure of the architect and his stagnant ideologies.

Key words: Tradiciones peruanas; architecture; ideology.

\section{Recibido: 08/11/2019 Aceptado: 08/12/2019}

\section{INTRODUCCIÓN}

En muchos países, se suele tener un concepto de la arquitectura como parte de la historia y de la cultura que se forma en cada nación. La sociedad ha visto en la arquitectura una herramienta para poder interpretar los ideales de cada obra planteada y su importancia en cada época relevante de la historia; de esta manera se puede analizar cómo las barreras que la arquitectura ha tenido no han sido tratadas, sino que se paró el desarrollo de ideas novedosas que estuvieran comprometidas con los valores cívicos, trayendo con ello un estancamiento de ideas y el fomento de barreras en la arquitectura.

En los edificios actuales, de acuerdo con Frank Gehry, «no hay sentido del diseño, ni respeto por la humanidad, por el buen criterio, ni por nada» (Sauras, 2014, párr. 5). Para el caso peruano, existen ambos problemas, concentrándose mucho más en las barreras de la arquitectura. Sin embargo, el estancamiento de ideas en el arquitecto peruano se ha visto muy presente en los últimos años, logrando así que la ciudad de Lima pasara el boom de la construcción bajo un gran margen de error. «Las tomas de tierras surgidas de iniciativas populares se hicieron de manera colectiva y organizada, con el respaldo tácito o explícito del Estado, creando inclusive distritos completos que hoy en día cuentan con más de 460 mil habitantes, como es Villa el Salvador» (García, Miyashiro, Rubio, Santa Cruz y Marces, 2009, pp. 228-229). 
Ahora bien, me parece que no solo en el creciente desarrollo de la sociedad peruana se presenta este problema de innovación de ideas y destrucción de barreras en la arquitectura, sino que también se hace presente en otras manifestaciones literarias del periodo. Para ser explícitos, creo que podemos reconocer este problema en uno de los productos más innovadores de la cultura literaria peruana: las tradiciones de Ricardo Palma.

En lo que sigue, analizaremos la manera en la que se desarrollan ambos problemas en «Al pie de la letra» $\mathrm{y}$ «Lavaplatos» de Ricardo Palma.

En un pasaje de «Al pie de la letra», el narrador describe a Paiva en los siguientes términos: «El hombre no entendía de acepción figurada en la frase. Había que ponerle los puntos sobre las íes» (Palma, 2015, p. 462).

Notemos la ironía en el uso de la expresión «Había que ponerle los puntos sobre las íes» para describir al protagonista. Esta frase significa "poner algo en claro», es decir, se describe al protagonista como alguien incapaz de poder procesar una idea, pues lleva todo a un plano literal, lo cual le proporcionaría distintos problemas en su vida profesional. Esto explicaría el motivo por el cual Paiva no es ascendido, tomarse las cosas de una forma literal provoca que se quede estancado en el rango que tiene en el Ejército. Esto se da a pesar de que él solo podía valer como un escuadrón entero, es decir, su rendimiento era excelente mientras no involucrara ninguna orden que tuviese que inferir. El eterno capitán Paiva no miraba más allá de una orden, las cosas eran como eran y no había cambios.

En este contexto podemos comparar al capitán con la figura actual del arquitecto peruano y los estudiantes de arquitectura, ya que son representados como profesionales que disponen de una gran imaginación y recursos; sin embargo, la situación actual no les permite avanzar. Es decir, la falta de figuras representativas de la 
arquitectura peruana, avances e innovaciones, falta de preocupación por desarrollar una arquitectura sostenible y funcional, sumada a la poca preocupación por el desarrollo de una buena enseñanza y compromiso con las obras que se desarrollan en el Perú, nos dan como resultado un estancamiento. Una prueba de ello sería uno de los edificios más peligrosos por su forma de construcción y acabado:

en la cuadra 9 de la avenida Abancay que parece superar toda ley de la física. Pese a contar con siete pisos, tiene un grosor lateral no mayor de 1,20 metros, donde no entraría ni una cama de dos plazas. Verlo desde afuera da vértigo, pero ingresar y subir hasta el último piso es casi una actividad de alto riesgo. No hay señalización ni implementos básicos de emergencia, y la salida está obstruida por comercios y cables de telefonía (León, 2017, párr. 1).

En este caso en particular observamos cómo la arquitectura peruana no avanza por falta de interés a la seguridad ciudadana y por una falta de culturización arquitectónica. El estancamiento aquí nace desde hace mucho tiempo. Entonces al referirnos a este, podemos enfocarnos en diversos aspectos, uno de ellos sería la falta de progreso, como se menciona en el texto: «Paiva era el capitán eterno. Para él no había más allá de los tres galoncitos» (Palma, 2015, p. 459).

Del fragmento citado llama mucho la atención que el narrador tenga una perspectiva muy pobre del protagonista a pesar de que este tiene suficiente potencial para ser más que un simple capitán. Además, menciona: "Para él no había más allá de tres galoncitos», dando a entender que el capitán se conformaba y no aspiraba a más. Situación que podríamos comparar con el país, ya que este a pesar de tener los suficientes recursos para superar la pobreza, simplemente decide quedarse estancado.

Bien, a pesar de que ambas comparaciones son racionales, no debemos olvidar que todo es provocado por algo, una de las causas 
del estancamiento sería la formación que se nos da desde nuestra casa de estudios. Muchas de las ideas estancadas que suelen llenar la cabeza de futuros profesionales y ya egresados, se pueden atribuir en parte al paro y deterioro del crecimiento de la arquitectura peruana. El arquitecto peruano puede ser comparado con esta tradición gracias a la falta de voluntad por querer mejorar y no poder hacerlo. Un ejemplo muy claro es la desmotivación que los egresados de la carrera tienen. $\mathrm{Al}$ avanzar en la carrera no solo notan que el país no invierte en su arquitectura, sino que, además, no todos tienen una visión de querer progresar y hacer un cambio para salir de esta situación paralizada que no aporta nada a nuestro país. Seguir todo al pie de la letra no solo provoca un paro de innovaciones, también nos detiene, ya que las bases de las que creemos que debemos partir, están mal. Esto es así porque nadie tiene el valor de desafiarlas e imponer una nueva «letra» que sea capaz de guiar a los futuros profesionales de la arquitectura. Si esta fuese desafiada, el apoyo a la persona que decide romper con esta ideología estancada, en muchos casos, no suele ser dado, provocando que se regrese al punto de donde partimos, donde los profesionales no tienen motivación por salir de esta paralizada ideología y todo sigue como siempre, dando una vuelta sin fin. Al hablar de la «letra» nos referimos a los conceptos básicos que creemos correctos.

Establecer un orden, o sea, «ir al pie de la letra», podría estar bien cuando hay una buena base que tomará en cuenta todos los factores que generarán un cambio al campo que se decide aplicar; sin embargo, ese no es el caso con los arquitectos peruanos, hay un estancamiento que más que ser un límite es una traba que no está siendo derribada. La arquitectura es la resolución de problemas, para hacer esto las opciones son infinitas, pero esto nos exige que los límites deben empezar a eliminarse o reducirse poco a poco, ello no solo permitirá un mejor acceso a ideas que creemos imposibles, también nos permitirá llegar a lo que siempre debió ser una 
prioridad: la evolución y renovación constante de la arquitectura peruana. Esto no solo permitirá cambiar ideales, a su vez, permitirá que todos vean lo que realmente es la arquitectura y cuánto puede influir en nuestras vidas en conjunto y de forma individual.

«La arquitectura cumple un rol importante como eje de desarrollo social [...] era primordial darle una mejor calidad de vida a los estudiantes» (Chávez, 2019, párr. 10). Los arquitectos comprometidos con su trabajo pueden llegar a superar la imagen del comandante Paiva en la que se encuentran encerrados. El compromiso y el entusiasmo suelen ser sentimientos que son puestos a prueba con mucha regularidad, pero la pasión por la carrera, que los estudiantes y profesionales eligieron o ya han elegido, debería ser inquebrantable como la bravura que tiene el capitán Paiva. Me parece que los errores siempre están en todo, pero no es sano concentrarse solo en lo malo, a veces lo más complicado puede ser lo más resaltante de algo. En el caso de la arquitectura, los trabajos más resaltantes son aquellos que toman una dificultad y la resaltan como la característica más innovadora del proyecto. Es por eso que el final de «Al pie de la letra» no me parece trágico, a pesar de que se da en función de una ideología errónea, dándonos a entender que todo tiene una consecuencia y que si no se trabaja en el problema, las ideologías estancadas que rodean a los arquitectos peruanos del siglo XXI pueden provocar inevitablemente un fracaso que afectará a toda la sociedad peruana; por lo que podemos tomar el final de esta narración como una protesta y aviso de lo que podría provocar no resolver los problemas de la sociedad peruana.

Cuando hablamos de barreras arquitectónicas nos referimos a la falta de cuidado en aspectos que involucran a toda la sociedad, un ejemplo claro puede ser cuando se deja de lado la construcción de rampas porque supuestamente «nadie» las usará. La tradición peruana del «Lavaplatos» nos menciona una situación: 
La hacienda de San Borja, en los alrededores de Lima, medía noventa y dos fanegadas de terreno, y como dotación de agua disfrutaba de ocho riegos y medio, lo que ciertamente era poquita cosa.

Los padres jesuitas, propietarios del fundo, decían que San Borja apenas tenía agua para que un pato nadase con holgura; pero ellos sabían ingeniarse para contar siempre con algunos riegos más a expensas de las haciendas vecinas, con cuyos dueños mantenían constantes litigios (Palma, 2015, p. 393).

No me parece coincidencia que los primeros párrafos de la lectura «Lavaplatos», de Ricardo Palma, empiecen con una queja sobre el problema de la falta de agua de San Borja, ya que nos expone uno de los más grandes problemas que había en la época colonial y que aún perdura a pesar del tiempo que ha pasado.

Como ya se ha definido antes, los arquitectos siempre han tenido como fin resolver los problemas de los usuarios para poder crear un espacio que tenga armonía entre la funcionalidad y un óptimo diseño, llevando un equilibrio. El caso en el Perú no es distinto, sin embargo, la arquitectura no siempre ha sido una prioridad para ayudar al crecimiento de las ciudades y pueblos del Perú. Tenemos diversos factores que alterarán y explicarán el porqué de las barreras que existen en la arquitectura y, a su vez, la razón por la cual se mantienen hasta el día de hoy.

Hace 100 años se inició la formación del arquitecto en el Perú, efectivamente mediante una ley, el Gobierno dispuso el 28 de abril de 1910 la creación de una Sección de Arquitectos Constructores en la entonces Escuela Nacional de Ingenieros, convertida en 1955 en la Universidad Nacional de Ingeniería, alma mater de la formación profesional tecnológica y arquitectónica peruana (Delgado, 2011, párr. 1). 
La arquitecta Delgado nos expone que antes de esta fecha (1910) la escuela de arquitectura del Perú no existía como un ente independiente, sino que estaba ligada a la ingeniería civil. Esta separación entre escuelas fue un comienzo para la cultura arquitectónica peruana del siglo XX. La sociedad de aquel siglo no tenía una preocupación por tener un crecimiento urbanístico óptimo. Las constantes crisis políticas y las migraciones dejaron en segundo plano diversos factores para un desarrollo adecuado del crecimiento de la sociedad.

Sin embargo, estos factores no justifican la falta de atención a los problemas de organización y distribución justa de los recursos que todos deberían tener. Con esto nos referimos a los recursos hidráulicos y la falta de atención a estos problemas, como expone la tradición peruana «Lavaplatos» o, sin ir muy lejos en el tiempo, podríamos hablar de cómo el gobierno no proporciona los recursos necesarios para vivir en un ambiente aceptable a distintos sectores del país. Dada nuestra gran diversidad de climas, el trato de cada área en el Perú debe ser especial y ajustarse a los factores que distinguen dicho lugar, es decir, no es lo mismo trabajar un edificio y acoplarlo a las necesidades del malecón de Chorrillos que uno ubicado en Puno. Todo aspecto considerado en el primer proyecto mencionado va cambiar dada la gran variación de necesidades y prioridades de los usuarios en cuestión.

En este punto del análisis, es pertinente preguntarse ¿por qué las barreras persisten en pleno siglo XXI? Y, por supuesto, ¿cómo se pueden eliminar? Si bien las barreras han persistido, debemos enfocarnos en cómo se trataban hace muchos años, siendo exactos, en el siglo XVII en la época colonial. El narrador nos señala lo siguiente:

Levantados de la mesa, no pudo el de Salvatierra dejar de manifestar su extrañeza por la grosería y desaseo en gente que, como los jesuitas, gozaba reputación de canta y limpia; pero el administrador de la hacienda se apresuró a contestar: 
- Harto nos duele, señor excelentísimo, la falta involuntaria en que hemos incurrido, y crea vuecencia que solo una absoluta imposibilidad nos ha impedido cambiar plato y cuchara para cada servicio.

— ¿Y qué imposibilidad puede ser esa, padre?

- Señor, la de que tenemos tan poca agua que no nos alcanza para hacer lavar platos.

El virrey no pudo dejar de sonreírse, y probablemente se dijo para sí: «Estos benditos varones no tienen puntada sin nudo, y cuando dan el ala es para mejor comerse la pechuga».

Y concluyó el de Salvatierra:

- Pues por si me ocurre volver a almorzar en San Borja, quiero evitar que los que me acompañen coman en plato sucio. Señor juez de aguas, asigne usía un riego más a esta hacienda para servicio de la cocina (Palma, 2015, p. 394).

No es menos que injustificada la solución del problema, a pesar de que se logró resolver la falta de agua, eso no justifica el porqué. Si analizamos, llegaremos a la conclusión de que solo se solucionó porque el virrey quería comer en platos limpios cuando visitase las haciendas. Entonces, tenemos a una autoridad de alto rango permitiendo que una barrera arquitectónica se derrumbe solo porque lo afecta directamente, ¿no es eso común hoy en día? Regresando a los siglos XX y XXI, tenemos distintos ejemplos de esto, los obstáculos arquitectónicos que proporcionan malos servicios a los usuarios no solo se dan por la falta de educación o ideas estancadas, también se deben a la falta de apoyo y preocupación por la arquitectura que nos rodea.

En Los siete saberes necesarios para la educación del futuro (Morin, 1999) se menciona la identidad terrenal como un problema que debe ser visto desde un punto de vista colectivo y no desde un punto de vista individual, ya que esto proporcionará una gama más amplia de soluciones. El proceso podría llevar más tiempo, pues se buscaría 
derribar las barreras de la arquitectura peruana, pero el resultado sería muy beneficioso para todos.

Los beneficios de este proceso no solo brindarían una mejor vista de la cultura arquitectónica peruana, también impulsarían a las futuras generaciones a preservar una imagen que más que de modo externo, nos debe impulsar a mejorar de forma interna, es decir, seguir innovando y desarrollar una arquitectura sin barreras. Los problemas y obstáculos siempre se generarán, puesto que son parte del avance en el tiempo y los cambios que se producen en un lugar. A pesar de eso, estar a la vanguardia de resolverlos es una de las metas que los profesionales y futuros profesionales de la arquitectura y todas las carreras deben tomar en cuenta y resolver en conjunto. Una arquitectura sin barreras, que cuide de todos los aspectos posibles, debe ser el principal objetivo de todos los implicados en esta carrera.

\section{CONCLUSIÓN}

En síntesis, las ideas estancadas y las barreras que rodean la arquitectura peruana son problemas que pueden ser resueltos luego de un profundo análisis, por lo que considero que las Tradiciones peruanas de Ricardo Palma: «Lavaplatos» y «Al pie de la letra» no solo nos invitan a reflexionar sobre diversos temas, sino que exponen de manera muy explícita las consecuencias y los motivos por los cuales la arquitectura no puede crecer dentro del país. Por ello debemos romper con los ideales paralizados que hemos formado a lo largo de los años y detener y comprender que para desarrollar una arquitectura sin barreras debemos tener un pensamiento colectivo. 


\section{REFERENCIAS}

Chávez, J. (2019). La historia de los arquitectos peruanos con el proyecto más premiado en el mundo. El Comercio. https:// elcomercio.pe/casa-y-mas/historia-detras-arquitectos-proyectoperuano-premiado-ano-mundo-ecpm-noticia-647919-noticia/

Delgado, C. (2011). 1910-2010. 100 años de arquitectura en el Perú. CICAP. Colegio de Arquitectos del Perú. Bitácora Arquitectura Peruana. http://www.arquitecturaperuana.pe/2011/01/1910-2010100-anos-de-arquitectura-en.html

García, R., Miyashiro, J., Rubio, D., Santa Cruz, P. y Marces, R. (2015). Desarrollo o crecimiento urbano en Lima: el caso de los distritos del Sur. En Perú hoy. Hacia otro desarrollo (pp. 223-247). Desco.

León, J. (2017). Terremoto en Lima: los edificios y casas que también están en alto riesgo. El Comercio. https:/elcomercio.pe/lima/ sucesos/terremoto-lima-edificios-capital-alto-riesgo-noticia460511-noticia/

Morin, E. (1999). Los siete saberes necesarios para la educación del futuro. Unesco.

Ortiz Partida, V. (2013). Belleza y funcionalidad en arquitectura. Iteso Magis https://magis.iteso.mx/content/belleza-y-funcionalidad-enarquitectura

Palma, R. (2015). Al pie de la letra y Lavaplatos. En Tradiciones peruanas. Séptima y octava series (M. Á. Rodríguez Rea, ed.; pp. 459-463, 393-394). Universidad Ricardo Palma, Editorial Universitaria.

Rudolph, P. (1993). Interview with Paul Rudolph. Interviewed by Robert Bruegmann. The Art Institute of Chicago. USA, New York.

Sauras, S. (2014). Sobre Frank Gehry-Yo, Icono. Cosas de arquitectos. https://www.cosasdearquitectos.com/2014/10/sobre-frank-gehryyo-icono/ 